\title{
Non-Hodgkings Lymphoma
}

\author{
Effiong Edet Bassey* \\ Department of Applied Microbiology and Brewing, Nnamdi Azikiwe University, Nigeria
}

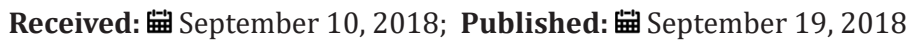

*Corresponding author: Effiong Edet Bassey, Department of Applied Microbiology and Brewing, Nnamdi Azikiwe University, Awka, Nigeria

\begin{abstract}
Lymphoma is the general term for cancers that develop in the lymphatic system. Lymphoma originates in developing lymphocytes (B-cells and T-cells), which have undergone a malignant change. They multiply without any proper order, forming tumors, which are collections of cancer cells. These tumors cause swelling in the lymph nodes and other parts of the body. Over time, malignant lymphocytes (called lymphoma cells) crowd out normal lymphocytes and eventually the immune system becomes weakened and can no longer function properly. This lymphoma affects the lymph nodes first and could also affect other lymphoid organs like spleen and bone marrow. The Non-Hodgkin's lymphoma can be classified into 2, depending on how fast or slow these tumors grow within the body namely; Aggressive non-Hodgkin's lymphoma and Indolent non- Hodgkin's lymphoma. Non-Hodgkin's lymphoma can also be classified based on the type of cells they emanate from namely; B-cells lymphoma and T-cells lymphoma. B-cell lymphoma are lymphomas that arise from developing B-cells, while T-cell lymphoma are lymphomas that arise from T -cells [1].

The causes of non-Hodgkin's lymphoma include infection like the Epstein-Barr virus infection in patients from specific geographic regions, exposure to certain chemicals ingredients in herbicides and pesticides such as organochlorine and phenoxy acid can lead to lymphoma and also immunosuppression. The extent to which the lymphoma has spread in body is called the stage of the disease. In stages 1 and 2, lymphoma is limited to one or two areas of the body (early stage). In stages 3 and 4 , the disease is more widespread in the body (advanced stage). It's signs and symptoms include; enlarged lymph node in the neck, fever, excessive sweating, unexplained fatigue, loss of appetite or weight loss. Non-Hodgkin's lymphoma is usually diagnosed by examining a lymph node biopsy specimen under a microscope using either core biopsy or surgical biopsy [2,3]. Non-Hodgkin's lymphoma can be treated using chemotherapy and radiation therapy as the two principal forms of treatment for NHL'. Stem cell transplantation can also be used for treating NonHodgkin's lymphoma. A healthy and nutritious diet is important to help your body cope with your disease and treatment.
\end{abstract}

\section{Introduction}

Non-Hodgkin lymphoma (NHL) is the term used for a diverse group of blood cancers that share a single characteristic-they arise from an injury to the DNA of a lymphocyte parent cell. The damage to the DNA is acquired (occurs after birth) rather than inherited. The altered DNA in one lymphocyte produces a malignant transformation. This transformation results in the uncontrolled and exaggerated growth of the lymphocyte. These lymphocytes and the formed cells have a better-than-normal chance to survive. The accumulation of those cells results in the tumor masses found in the lymph nodes and other sites in the body. Lymphoma is a general term for a group of blood cancers that start in the lymphatic system. About 54 percent of the blood cancers that occur each year are types of lymphomas. Lymphoma results when a lymphocyte (a type of white blood cell) undergoes a malignant change and multiplies, eventually crowding out healthy cells and creating tumors. These

tumors enlarge the lymph nodes and/or grow in other sites that are part of the immune system (for example, the skin and other organs). Lymphocytic leukemia, a blood cancer that also originates in a lymphocyte, is closely related to lymphoma [4].There are two major types of lymphoma: Hodgkin lymphoma and non-Hodgkin lymphoma (NHL). More than 60 specific non-Hodgkin lymphomata (NHL) subtypes have been identified.

These have been assigned "diagnostic designations" (names) by the World Health Organization (WHO) in its Classification of Tumors: Tumors of Hematopoietic and Lymphoid Diseases (WHO 2008). Oncologists (cancer specialists) further characterize the NHL subtypes according to how fast (aggressive) or slow (indolent) the disease progresses (National Cancer Institute, 2017).The subtype of NHL (and whether it is the indolent form or the aggressive form) determines appropriate treatment, so getting an accurate diagnosis 
is very important. Over time, malignant lymphocytes (called lymphoma cells) crowd out normal lymphocytes and eventually the immune system becomes weakened and can no longer function properly. NHL generally develops in the lymph nodes or in lymphatic tissue found in organs such as the stomach, intestines or skin. In some cases, NHL involves marrow (spongy tissue in the hollow central cavity of the bones that is the site of blood cell formation) and blood (National Cancer Institute, 2007). Lymphoma cells may develop in one or many sites in the body. In lymphoma, the majority of these cancerous cells are found in the lymphatic system but can also be found in other areas of the body.

\section{The Cells That Form Lymphoma}

\section{Normal Blood and Marrow and the Lymphatic System}

Blood and Marrow: Blood is composed of plasma and cells suspended in plasma. Plasma is made up of water in which many chemicals are dissolved. These chemicals include

a) Albumin, the most common protein in blood

b) Blood-clotting proteins made by the liver

c) Erythropoietin, a protein made by the kidneys that stimulatesred blood cell production

d) Immunoglobulins, antibodies made by plasma cells in response to infections including those we develop from our vaccinations (such as poliovirus antibodies, which are made by normal plasma cells in the bone marrow)

e) Hormones (such as thyroid hormone and cortisol)

f) Minerals (such as iron and magnesium)

g) Vitamins (such as folate and vitamin B12)

h) Electrolytes (such as calcium, potassium and sodium)

i) Antibodies, which are made by plasma cells [5].

j) The cell suspended in plasma include red blood cells, platelets and white blood cells (neutrophils, monocytes, eosinophils, basophils, and lymphocytes).

k) The red blood cells make up a little less than half the volume of the blood. They are filled with hemoglobin, the protein that picks up oxygen in the lungs and delivers it to the ells all around the body; hemoglobin then picks up carbon dioxide from the body's cells and delivers it back to the lungs, where it is removed when we exhale.

\section{The Platelets}

The Plates are mall cells (one-tenth the size of red blood cells) that help stop bleeding at the site of an injury in the body. For example, when a person has a cut, the vessels that carry blood are torn open. Platelets stick to the torn surface of the vessel, clump together, and plug up the bleeding site with the help of blood- clotting proteins such as fibrin, and electrolytes such calcium. Later, a firm clot form. The vessel wall then heals at the site of the clot and returns to its normal State.

\section{The Neutrophils and Monocytes}

These are white blood cells. They are called "phagocytes" (eating cells) because they can ingest bacteria or fungi and kill them. Unlike the red blood cells and platelets, the monocytes can leave the blood and enter the tissue, where they can attack the invading organisms and help combat infection. Eosinophils and basophils are types of white blood cells that respond to allergens or parasites. Most lymphocytes, another type of white blood cell, are found in the lymph nodes, the spleen and the lymphatic channels, but some enter the blood. There are three major types of Imphocytes: Tlymphocytes (T cells), B lymphocytes (B cells) and natural killer (NK) cells. These cells are a key part of the immune system.

\section{Bone Marrow}

Bone Marrow is a spongy tissue where blood cell development takes place. It occupies the central cavity of bones. In newborns, all bones have active marrow. By the time a person reaches young adulthood, the bones of the hands, feet, arms and legs no longer have functioning marrow. The spine (vertebrae), hip and shoulder bones, ribs, breastbone and skull contain the marrow that makes blood cells in adults. The process of blood cell formation is called "hematopoiesis," A small group of cells, the stem cells, develop into all the blood cells in the marrow by the process of differentiation [5]. In healthy individuals, there are enough stem cells to keep producing new blood cells continuously. Blood passes through the marrow and picks up the fully developed and functional red and white blood cells and platelets for circulation in the blood.

Some stem cells enter the blood and circulate. They are present in such small numbers that they cannot be counted or identified by standard blood count tests. Their presence in the blood is important because they can be collected by a special technique. There are also methods to induce more stem cells to leave their home in the marrow and circulate in the blood, allowing a greater stem cell collection to occur. If enough stem cells are harvested from a compatible donor, they can be transplanted into a recipient. Stem cell circulation, from marrow to blood and back, also occurs in the fetus. After birth, placental and umbilical cord blood can be collected, stored and used as a source of stem cells for transplantation [6].

\section{The Lymphatic System}

The lymphatic system is made up of a vast network of lymph vessels, similar to blood vessels that branch out into all the tissues of the body. The two main roles of the lymphatic system are: to drain excess fluid from the body's tissues, filter it and return it to the blood stream; and to hour specialist white blood cells -lymphocytes -to help fight infection. There are two types lymphocytes, B-cells and T-cells. These cells protect us by destroying harmful microorganisms such as bacteria and viruses. As such, the lymphatic system forms 
part of the immune system, which protects our bodies against disease and infection.

\section{Causes and Risk Factors of Lymphoma}

\section{Causes and Risk Factors}

The age-adjusted incidence of NHL rose by more than 89.5 percent from 1975 to 2010, an average annual increase of about 2.6 percent. The reasons for this increase are not certain, and there are probably multiple causes. Since the mid-1980s, the incidence of in individuals with the human immunodeficiency virus (HIV) or acquired immune deficiency syndrome (AIDS) has contributed modestly to the overall increase in incidence. However, the increase in the general population began before the spread of HIV. Furthermore, while NHL is between 50 and 100 times more prevalent among people with HIV/AIDS than among uninfected individuals, newer therapies for HIV infection have lowered the incidence of AIDS-related lymphoma [1]. There is a higher incidence of NHL in farming communities. Studies suggest that specific ingredients in herbicides and pesticides such as organochlorine, organophosphate and phenoxyl acid compounds are linked to lymphoma. The number of lymphoma cases caused by such exposures has not been determined. Other studies suggest that pesticides used in agricultural, commercial and home and garden application are associated with cancer risk [1].

\section{Infection}

a. Epstein-Barr virus (EBV) infection-in patients from specific geographic regions-is strongly associated with African Burkitt lymphoma. The role of the virus is unclear, since African Burkitt lymphoma also occurs among people who have not been infected with EBV. Epstein-Barr virus infection may play a role in the increased risk of NHL in persons whose immune systems are suppressed as a result of organ transplantation and its associated therapy [4].

b. Human T-lymphotropic virus (HTLV) is associated with a type of T-cell lymphoma in patients from certain geographic regions in southern Japan, the Caribbean, South America and Africa.

c. The bacterium Helicobacter pylori causes ulcers in the stomach and is associated with the development of mucosaassociated lymphoid tissue (MALT) lymphoma in the stomach wall.

Immunosuppression: A small percentage of lymphomas occur in people whose immune system has been weakened (immunosuppressed) either by a viral infection such as human immunodeficiency virus (HIV) or as a result of drugs which affects the function of the immune system (immune-suppressants). These drugs are commonly used when someone has a heart, lung or other organ transplant [7].
Chemicals: Some evidence suggests that farmers and other people who are exposed to high concentrations of agricultural chemicals such as pesticides and fertilizers may have a higher risk of developing lymphoma.

Lifestyle: Certain lifestyle factors appear to increase the risk of NHL including smoking (follicular lymphoma) and obesity (diffuse large B-cell lymphoma).

\section{Signs and Symptoms of Lymphoma}

a. Enlarged lymph nodes can result from inflammation and are not necessarily a sign of cancer. However, if enlarged lymph nodes are detected during a physical examination or during an imaging test (for example, a chest $x$-ray) and there is no obvious explanation such as a nearby infection, lymphoma could be the cause.

b. Occasionally, the disease starts in a site other than the lymph nodes, such as a bone, a lung, the gastrointestinal tract or the skin. In these circumstances, patients may experience symptoms that are associated with that site, such as bone pain, cough, chest pain, abdominal pain, rashes or skin.

c. Patients may also have fever, excessive sweating (especially noticeable at night), unexplained fatigue, loss of appetite or weight loss. During a medical examination, the doctor may detect an enlarged spleen. Sometimes, a person has no symptoms and the disease may only be discovered during a routine medical examination or while the person is under care for an unrelated condition [8].

\section{Diagnosis of Lymphoma}

Diagnosis is of NHL is usually made by examining a lymph node biopsy specimen (piece of node taken and studied under the microscope); the examination includes tests like lymph node biopsy, Flow Cytometry, Bone Marrow aspirator and Physical Examination [9].

Lymph Node Biopsy: Making an accurate diagnosis of the specific type of NHL that a patient has can be difficult. Since the subtypes of NHL can be confused with one another, and the prognosis (likely course of a disease), treatment goals and treatment approach may be different, a precise diagnosis is needed. It requires an experienced hematopathology's analyze the biopsy slides. Here, all or part of the lymph nodes are removed by the doctor, and then view the tissue under a microscope to look for cancer cells. The following are different types of biopsies that can be done.

Core or Fine Needle Biopsy: involves inserting a needle into a lymph node or lump and removing a sample of tissue [2].

Surgical or Excision Biopsy: involves removing the entire swollen lymph node. 
Laparoscopy: Laparoscopy is a surgical procedure in which a narrow tube with a tiny microscope attached is inserted through a small cut in the skin. It allows the surgeon to see the inside of your abdomen pelvis, and to take a biopsy if needed. This is done under general an aesthetic and you will have a few stitches afterwards.

Flow Cytometry: This is a laboratory test that measures the number of cells in a sample, the percentage of living cells in a sample, and also the shape, size and presence of tumor markers on the cell surface. The cells are stained with a light sensitive dye, placed in a fluid, and passed in a stream before a laser. The measurements are based on how the light-sensitive dye reacts to the light.

Bone Marrow Aspiration: This is the removal of bone marrow and a small piece of bone by inserting a needle into the hipbone or breastbone. A pathologist views the bone under a microscope to look for signs of cancer.

Physical Examination: An examination of the body to check general Signs of health, including checking for signs of disease, such as lumps. A history of the patient's health habits and past illnesses and treatments will also be taken.

\section{Treatment of Non-Hodgkins Lymphoma}

The Non-Hodgkins Lymphoma can be treated through the use of Various therapies. Treatment for lymphomas may involve the use of chemotherapy" radiotherapy, stem cell transplant, Immunotherapy, targeted therapy and antibiotics therapy (National Cancer Institute, 2017). Many low-grade lymphomas take years to grow, cause few (if any) symptoms, and do not necessarily need to be treated on diagnosis. In these cases, the doctor may recommend regular checkups to carefully monitor your lymphoma and general health. If the lymphoma is limited to a small group of lymph nodes (stage 1 or 2), radiotherapy alone may be able to cure some low-grade lymphomas or control them for a long time [10]. If the low-grade lymphoma is more widespread in the body, the decision to treat the disease or not depends on a number of factors such as: where the lymphoma has spread to, how large the areas of lymphoma are, if you are experiencing any symptoms of your lymphoma, and your general health [11].

\section{Factors that Influence the Treatment of Non- Hodgkins Lymphoma}

The most effective treatment plan for a patient with NHL is individualized and depends on

a. The subtype of NHL (knowing whether the lymphoma cells are most closely related to $\mathrm{T}$ cells, B cells or natural killer [NK] cells gives the doctor important clues as to which treatments should be used).

b. The stage and category of the disease (the distribution of lymphoma throughout the body is sometimes important in forming decisions about treatment; c. Factors such as fever, drenching night sweats and weight loss of more than 10 percent of body weight, referred to as "B symptoms"

d. The presence of lymphoma in areas of the body outside of the lymph nodes (extranidal involvement).

\section{Conclusion and Recommendation}

Non hodgkins lymphoma has been seen as one of the most wide spread disease in Australia. It affects all age ranges especially between the ages of 40 to 60 . The true causes of Non-Hoggkins lymphoma includes Exposure to chemicals, intake of immunesuppressant drugs, Epstein-Barr virus infection and inherited autoimmune diseases. Tests like flow cytornetry, lymph biopsy and bone marrow aspiration are used for diagnosing Non-Hodgkins lymphoma. In conclusion, the treatment of Hodgkins lymphoma has majorly impacted the care of patients with other types of cancer. The High cure rate in this disease represents one of the biggest victories in medical and radiation oncology. A healthy and nutritious diet is important to help your body cope with your disease and treatment. Conclusively, there are a variety of programs designed to help ease the emotional and financial strain associated with cancer and its treatment for example the leukaemia foundation.

\section{References}

1. Alavanja MC, Ross MK, Bonner MR (2013) Increased cancer burden among pesticide applicators and others due to pesticide exposure: A Cancer Journal for Clinicians 63(2): 120-142.

2. Barrington SF, Hutchings M, Meignan M (2014) Role of Imaging in the Staging and B-cell lymphoma in younger patients: current strategies and future directions. Onco Targets and Therapy 6(4): 289-291.

3. Benjamin E, Anderson FW (2012) Adult Non-Hodgkins lymphoma. Journal of the National Cancer Institute 104 (102): 888-915.

4. Cheson BD (2015) Staging and Response assessment in Lymphomas the Chinese Clinical 40(1): 5.

5. Dong L, Chen, Y, Masaki Y (2013) Possible Mechanisms of lymphoma development in Emission Tomography/Computerized Tomography in malignant lymphoma. Japanese Journal of Clinical Hematology 57(10): 2008-2012.

6. Greenlee RT, Murray T, Bolden, S, Wingo PA (2010) Cancer Statistics. Cancer Journal Hodgkin's lymphoma. Seminar Haematology 30: 286296.

7. Karlin P, Coiffier B (2013) Improving survival and preventing recurrence of diffuse large lymphoma. Current Opinion in Oncology 24(5): 461-465.

8. Marchese, Lange (2010) Effects of Physical therapy intervention for children with acute lymphoblastic Leukaemia. Pediatric Blood Cancer 42(2): 127-133.

9. Michallet AS, Lebras, L, Coiffier B (2012) Maintenance therapy in diffuse large B-cell Oncology 22(4): 2304-3865.

10. Rabkin CS, Devesa SS, Zahm SH, Gail MH (2011) Increasing incidence of non-Response assessment of Lymphoma. Journal of Clinical Oncology 32(27): 3048-3058.

11. Terauchi T, Ketsueki R (2016) Update on the use of fluorodeoxyglucoseposition 1: 2-3. 
(c) (P) This work is licensed under Creative

To Submit Your Article Click Here:

Submit Article

DOI: 10.32474/IPDOAJ.2018.02.000130

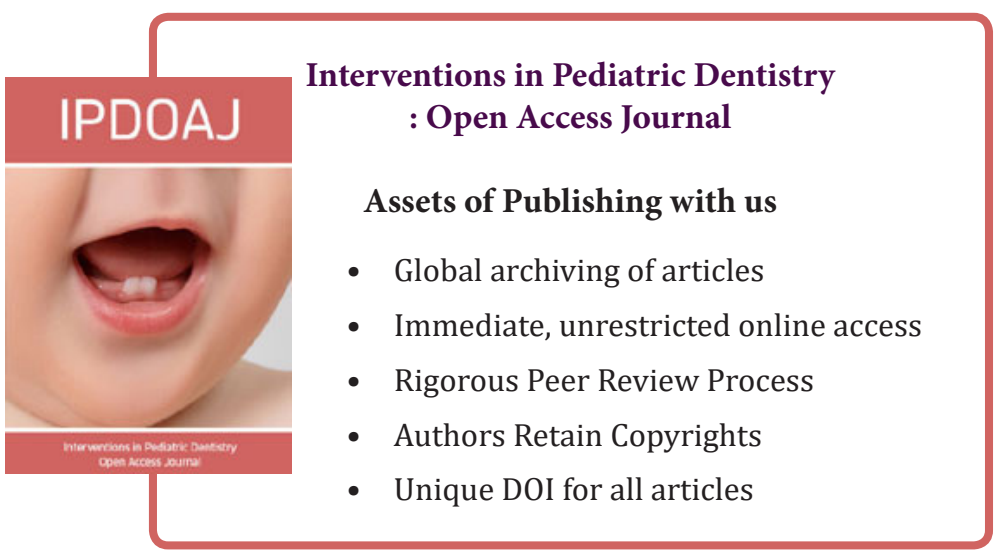

